Ota Konrád

\title{
Widersprüchlich und unvollendet
}

Die Demokratie der Ersten Tschechoslowakischen Republik 1918 bis 1938

\section{Die Tschechoslowakei der Zwischenkriegszeit als ein Vorbild?}

In dem Roman „Taschenführer einer intelligenten Frau durch ihr eigenes Schicksal“ zeichnete der im US-amerikanischen Exil lebende tschechische Journalist Pavel Tigrid folgendes Bild der sogenannten Ersten Tschechoslowakischen Republik:

„[... zivilisierte Beziehungen zwischen den Leuten, gemeinsam anerkannte Hierarchie der Werte, bescheidenes aber unnachgiebiges nationales Selbstbewusstsein, Achtung der Tradition, der ehrlichen Arbeit als auch des ehrlichen Worts, Unterstützung für diejenigen, die wir gewählt hatten, gute Schulen, saubere Züge sowie Restaurants mit sauberen Tischtüchern und mit netter Bedienung ... Das alles war in der Ersten Republik - sicherlich nicht in einem hinreichenden und ausgewogenen Ausmaß - vorhanden, aber es war vorhanden."1

Obwohl dieses Bild vor allem den Lebensstil der mittleren und oberen Schichten der tschechoslowakischen Gesellschaft wiedergibt, ${ }^{2}$ wurde es nach der Wende zur fast ausschließlichen Optik, durch die an die Zwischenkriegszeit erinnert wurde. Der erste Präsident nach 1989, Václav Havel, griff nicht nur in seinen Reden auf dieses Erbe zurück. So sollten umfangreiche Renovierungsarbeiten die in den Zeiten des Kommunismus verwahrloste Prager Burg (den traditionellen Sitz des Präsidenten) wieder im alten Glanz erstrahlen lassen - ähnlich wie auch Tomáš Garrigue Masaryk die in den Zeiten der Habsburgermonarchie vernachlässigte Burg nach der Gründung der Tschechoslowakei hatte umbauen lassen. Renoviert werden mussten nach 1989 jedoch nicht nur die Gebäude: Die ganze Gesellschaft sollte wieder in die natürliche Bahn ihrer Geschichte zurückkehren, aus welcher sie durch den schändlichen „Kommunismus“ herausgerissen worden war. Dieser Kurs sollte zurück nach Europa, zu Freiheit, Selbstständigkeit, Demokratie und zu Wohlstand führen. Die Erste Tschechoslowakische Republik diente hier als Vorbild und Wegweiser.

Bald zeigte sich jedoch die Widersprüchlichkeit dieser Tradition: Mit der Spaltung der Tschechoslowakei 1992 und den angespannten Beziehungen zu Deutschland wegen der sudetendeutschen Frage kam auch der problematische Teil des Erbes der Ersten Republik zum Vorschein: die Beziehungen zwischen beiden

\footnotetext{
${ }^{1}$ Pavel Tigrid, Kapesní průvodce inteligentní ženy po vlastním osudu, Prag ${ }^{2} 1990$, S. 276.

${ }^{2}$ Vgl. Stanislav Holubec, A „Golden Twenty Years“, or a Bad Stepmother? Czech Communist and Post-Communist Narratives on Everyday Life in Interwar Czechoslovakia, in: Acta Poloniae Historica 110 (2014), S. 23-48, hier S. 38.
} 
staatstragenden Nationen, den Tschechen und Slowaken - in der Zwischenkriegszeit offiziell als die „Tschechoslowaken“ wahrgenommen - als auch zu den nationalen Minderheiten. Neben der Literatur, die die Erste Republik durch eine rosarote Brille sah, entstand auch kritische fachhistorische Literatur. ${ }^{3}$

Nichtsdestoweniger beruhigte sich ungefähr nach der Jahrtausendwende die Diskussion. Die Erste Republik wurde schrittweise historisiert und in der öffentlichen Erinnerung zu einem zwar wichtigen, doch zumeist schon entkonkretisierten Symbol. Die Geschichtspolitik wurde formalisiert und ritualisiert. Dies ist wichtig, denn bis heute verkörpert die Erste Republik grundlegende Werte wie Staatlichkeit, Selbstständigkeit, Demokratie und Wohlstand, und nur diesem Umstand ist es zu verdanken, dass die Erinnerung an ihre Gründung immer noch als der wichtigste Staatsfeiertag funktionieren kann. Das Erbe der Ersten Republik war und ist nämlich höchst widersprüchlich, ${ }^{4}$ ähnlich wie es widersprüchlich ist, dass sich der heutige tschechische Staat, welcher hinsichtlich der Grenzen, ethnischen Zusammensetzung und internationalen Stellung nur wenig mit der Tschechoslowakei der Zwischenkriegszeit gemeinsam hat, noch immer klar zu ihr bekennt.

Von diesem Gesichtspunkt werde ich die tschechoslowakische Staatsgründung betrachten. Dabei konzentriere ich mich auf den Nationsbegriff als Mittel zum „Aufbau des Staats“. ${ }^{5}$ Im Falle der Tschechoslowakei, die sich für einen Sieger des Ersten Weltkriegs hielt, hatte er eine konstitutive Kraft, zugleich jedoch rief er viele, unvorhersehbare innere Spannungen und Widersprüche hervor und konnte ganz gegensätzlich gedeutet werden. Diese innere Widersprüchlichkeit war für den Staat prägend. Soll die Zwischenkriegszeit wieder zum Gegenstand einer kritischen historischen Forschung als auch einer kritischen öffentlichen Erinnerung werden, so muss diese Widersprüchlichkeit ernst genommen werden.

\footnotetext{
${ }^{3}$ Vgl. Jaroslav Kučera, Minderheit im Nationalstaat. Die Sprachenfrage in den tschechischdeutschen Beziehungen 1918-1938, München 1999. Einen Überblick über die Literatur zur Geschichte der Tschechoslowakei in der Zwischenkriegszeit liefert die Studie von Ines Koeltzsch/ Ota Konrád, From „Islands of Democracy“ to „Transnational Border Spaces“. State of the Art and Perspectives of the Historiography on the First Czechoslovak Republic since 1989, in: Bohemia 56 (2016), S. 285-327.

${ }^{4}$ Bis auf die oben erwähnte kurze Periode der 1990er Jahren war das Bild der Tschechoslowakei der Zwischenkriegszeit uneindeutig. Schon unmittelbar kurz nach 1945 knüpfte man nicht nur an sie an, sondern sie wurde zugleich zum Objekt der Kritik. Aus den vermeintlichen Fehlern dieses Staats (das zersplitterte parteipolitische System, ,zu große“ Zugeständnisse an die nationalen Minoritäten) sollte die neue Tschechoslowakei nach 1945 lernen. Nur ein paar Jahre später, nach der kommunistischen Machtübernahme im Februar 1948, wurde sie sogar zu einem Gegen- und Feindbild.

${ }^{5}$ So lautet der bekannte Titel eines Buchs über die Gründungsjahre der Tschechoslowakei aus der Feder des Journalisten Ferdinand Peroutka, Budování státu. Československá politika v letech převratových [Aufbau des Staats. Die tschechoslowakische Politik in den Umbruchsjahren], 4 Bde., Prag 1933-1936.
} 


\section{Im „zivilisierten Westen“}

In der erstmals 1918 publizierten Studie „Neues Europa. Der slawische Standpunkt“ deutete Tomáš Garrigue Masaryk den Krieg als einen Kampf zwischen der „mittelalterlichen“ Theokratie der Zentralmächte auf der einen Seite und den modernen, durch die Entente-Staaten verkörperten Ideen wie Demokratie oder Gewissensfreiheit auf der anderen. Dem „pangermanischen“ Griff nach der Herrschaft sollte ein Band freier und demokratischer Nationalstaaten von der Ostsee bis zum Schwarzen und zum Adriatischen Meer Einhalt gebieten. ${ }^{6}$

Aus dieser Perspektive schien die Gründung der Tschechoslowakei im Einklang mit der geschichtlichen Entwicklung zu stehen. Der neue Staat avancierte als eine Art „Agent des zivilisierten und demokratischen Westens“ im Osten zum Garanten der neuen Ordnung in der Region. Zu seinem Gegen- und Feindbild wurden die alten „destruktiven Kräfte“, denen die „konstruktiven“, wiederaufbauenden Kräfte des neuen Staats gegenübergestellt wurden. ${ }^{7}$ Dadurch bekam das tschechoslowakische nationale Narrativ eine starke fortschrittliche Dimension, die die Selbstwahrnehmung und Selbststilisierung des Staats sowohl damals als auch später erheblich geprägt hat.

Dem tschechoslowakischen Narrativ war jedoch auch eine innere Widersprüchlichkeit eigen. Dieses nationale Siegesnarrativ musste stets vom selbst konstruierten „Westen“ als solches bestätigt werden. ${ }^{8}$ Wenn es dazu nicht kam, geriet dieses Narrativ in eine Schieflage. Vieles davon konnte man durch geeignete Strategien verwischen: Sowohl die genaue Summe der „Befreiungsgebühren“, also de facto der Reparationen, die die Tschechoslowakei den Entente-Staaten bezahlen sollte, als auch der Anteil Prags an den Kriegsschulden der Monarchie - die nur schwer mit dem beanspruchten Siegerstatus in Einklang zu bringen waren - wurden lange Zeit geheim gehalten. Und trotz der komplizierten Verhandlungen im Vorfeld der Locarno-Konferenz von 1925, ihres für die Tschechoslowakei zwiespältigen Ergebnisses und der untergeordneten Stellung von Außenminister Edvard Beneš bei der Tagung, erklärte er Locarno im Nachhinein zu einem eindeutigen Erfolg der tschechoslowakischen konstruktiven europäischen Politik. Auf dem in der Tschechoslowakei kolportierten Foto der Tagungsteilnehmer erschien er folglich als Gleicher unter Gleichen. ${ }^{9}$

\footnotetext{
${ }^{6}$ Vgl. die aktuelle kritische Edition von Tomáš Garrigue Masaryk, Nová Evropa. Stanovisko slovanské [Das neue Europa. Der slawische Standpunkt], Prag 2016.

${ }^{7}$ Eva Hajdinová/Ota Konrád/Jana Malínská (Hrsg.), Edvard Beneš, Němci a Německo. Edice dokumentů [Edvard Beně̌, Deutsche und Deutschland. Dokumentenedition], Bd. II/1: 1919-1928, Prag 2015, Dok. 131, S. 314.

${ }^{8}$ Vgl. Jakub Eberle, Desire as Geopolitics. Reading the Glass Room as Central European Fantasy, in: International Political Sociology (im Druck).

${ }^{9}$ Zur Tschechoslowakei und der Konferenz vgl. Radko Břach, Die Tschechoslowakei und Locarno. Europäische Variationen, München 2011.
} 
Doch in einem Fall konnte diese Strategie nicht erfolgreich sein: Im September 1938 wurde das bisherige tschechoslowakische Narrativ radikal in Frage gestellt. ${ }^{10}$ Daher stellt das Münchner Abkommen die tschechische nationale Niederlage schlechthin dar und ist als „Verrat des Westens“ mit vielen Emotionen verbunden. ${ }^{11}$ So schrieb schon 1938 der tschechische Dichter František Halas in dem Gedicht „Das Lied der Beklemmung“: „Es läutet, läutet die Glocke des Verrats / Und klingt durch wessen Hand? / Süßes Frankreich, stolzes Albion / Wir haben sie geliebt, doch nicht gekannt!“12

Der Versuch, den tschechoslowakischen Staat und die tschechoslowakische Nation auf der mental map des „zivilisierten Westens“ zu verorten, war jedoch nicht nur auf die westeuropäischen Verbündeten begrenzt. Die globalen Exportinteressen, die vom Staat unterstützten und auf den „Orient“ ausgerichteten wissenschaftlichen Expertisen oder der Ruf nach einem stärkeren Engagement außerhalb von Europa, lassen den neuen Staat als einen vorwärtsstrebenden, westlichen erscheinen. ${ }^{13}$ Dies galt nicht nur für die globalen Unternehmen, wie zum Beispiel den Schuhproduzenten Bata ${ }^{14}$ oder die Maschinenbaufirma Škoda, sondern auch für einen bedeutenden Teil der kulturellen Eliten, die die Welt für das einheimische Publikum öffneten und zugleich den eigenen Staat in dieser Welt als einen fortschrittlichen darzustellen versuchten. Eine solche globale Sicht bestritt die Existenz der Nation nicht; sie ließ sie jedoch in einem anderen Licht erscheinen und gab ihr einen anderen Inhalt, als dies aus einer nur lokalen Perspektive möglich gewesen wäre.

Diese Sicht war auch eng mit dem inneren „Aufbau“ eines zuerst sehr heterogenen Staats verknüpft. Für eine solche „innere“ Kolonisierung setzten sich nämlich oft die gleichen Personenkreise und Institutionen ein, die sich auch für die Ansiedlung der Tschechoslowaken in fernen Ländern stark machten. ${ }^{15}$ Sie ließen auch in diesem Fall die Tschechoslowaken als eine fortschrittliche, reife Nation erscheinen, die das Licht der Zivilisation bis in die kleinsten und rückständigsten Dörfer bringt. Die im Laufe der Erschließung und Integration der oft als exotisch

\footnotetext{
${ }^{10}$ Aus der schon unüberschaubaren Fülle der Literatur zum Münchner Abkommen und zur Appeasement-Politik vgl. Vít Smetana, Ani vojna, ani mír. Velmoci, Československo a střední Evropa v sedmi dramatech na prahu druhé světové a studené války [Weder Krieg noch Frieden. Großmächte, Tschechoslowakei und Zentraleuropa in sieben Dramen an der Schwelle des Zweiten Weltkriegs und des Kalten Kriegs], Prag 2016, hier S. 18-75, und Jürgen Zarusky/ Martin Zückert (Hrsg.), Das Münchener Abkommen von 1938 in europäischer Perspektive, München 2013.

${ }^{11}$ Zum tschechischen Trauma des Münchner Abkommens vgl. Jan Tesař, Mnichovský komplex. Jeho př́ičiny a důsledky [Münchner Kompex. Seine Ursachen und Folgen], Prag 22014.

${ }^{12}$ František Halas, Zpěv úzkosti, in: Ders., Torzo naděje [Torso einer Hoffnung], Prag ${ }^{3} 1980$, S. 21-24, hier S. 22.

${ }^{13}$ Vgl. Sarah Lemmen, Locating the Nation in a Globalizing World. Debates on the Global Position of Interwar Czechoslovakia, in: Bohemia 56 (2016), S. 456-473, hier S. 472.

${ }^{14}$ Vgl. Martin Jemelka/Ondřej Ševeček, Tovární města Batova koncernu. Evropská kapitola globální expanze [Fabrikstädte des Bata-Konzerns. Europäisches Kapitel einer globalen Expansion], Prag 2016.

15 Vgl. Lemmen, Locating, S. 469.
} 
wahrgenommenen Ostteile der neuen Republik ${ }^{16}$ zur Schau getragene neue Identität der Tschechoslowaken konnte dabei auch die Mitglieder der ethnischen Minoritäten inkludieren. ${ }^{17}$

Neben diesen Tendenzen, die außer von technischen und kaufmännischen Eliten, auch von liberalen, städtischen Literaten, darunter vielen jüdischen Autoren, ${ }^{18}$ vorangetrieben wurden, traten jedoch auch zunehmend gegensätzliche Trends auf, die die Rückkehr zum Lokalen, zu den eigenen nationalen Wurzeln - oft in Form agrarromantischer Vorstellungen - forderten. Dies galt auch für die Deutschen in der Tschechoslowakei, bei denen beispielsweise die deutschen Historiker, Germanisten oder Volkskundler die Rückbesinnung auf die eigenen „Volkswurzeln“ als Mittel der nationalen Erneuerung und engen Verbindung mit dem „ganzen“ deutschen Volk propagierten. ${ }^{19}$ Diese oft gegensätzlichen Perspektiven, die eine unterschiedliche Auslegung des Nationsbegriffs nach sich zogen, spiegelten sich auch in Politik, Kultur und Mentalitäten der Tschechoslowakei der Zwischenkriegszeit wider und erzeugten erhebliche Kluften.

\section{Die Nation und die Stabilisierung nach innen}

Wie schon angedeutet, spielte die Vorstellung einer siegreichen Nation, welche nach 1918 die tschechische Wahrnehmung prägte, eine große Rolle auch hinsichtlich der Stabilisierung des Staats nach innen. Nach dem Ersten Weltkrieg lag Zentraleuropa in Trümmern. Die „Kultur der Niederlage“20 ließ nach dem verheerenden Krieg den Raum offen für eine radikale Infragestellung der bisherigen Ordnung. Die Nachkriegsgewalt in Deutschland und Ungarn und der Bürgerkrieg auf dem Gebiet des ehemaligen Romanow-Imperiums erschwerten die Nachkriegsrekonstruktion beziehungsweise machten sie fast unmöglich. ${ }^{21}$ Die neuen Republiken wie Deutschland oder Österreich standen auf äußerst fragilen Fundamenten und waren von Anfang an mit zahlreichen Hypotheken belastet.

\footnotetext{
${ }^{16}$ Vgl. Stanislav Holubec, „We bring order, discipline, Western European democracy, and culture to this Land of former oriental chaos and disorder". Czech Perceptions of Sub-Carpathian Rus and its Modernisation in the 1920s, in: Joachim von Puttkamer/Wlodzimerz Borodziej/ Stanislav Holubec (Hrsg.), Mastery and Lost Illusions. Space and Time in the Modernization of Eastern and Central Europe, München 2014, S. 223-250.

${ }^{17}$ Vgl. Felix Jeschke, „Mountain Men“ on „Iron Horses“. National Space in the Representations of New Railway Lines in Interwar Czechoslovakia, in: Bohemia 56 (2016), S. 437-455, hier S. 451.

${ }^{18}$ Vgl. Ines Koeltzsch, Geteilte Kulturen. Eine Geschichte der tschechisch-jüdisch-deutschen Beziehungen in Prag (1918-1938), München 2012, S. 179-251.

${ }^{19}$ Vgl. Petr Lozoviuk, Interethnik im Wissenschaftsprozess. Deutschsprachige Volkskunde in Böhmen und ihre gesellschaftlichen Auswirkungen, Leipzig 2008, und Ota Konrád, Dějepisectví, germanistika a slavistika na Německé univerzitě v Praze 1918-1945 [Geschichtsschreibung, Germanistik und Slawistik an der Deutschen Universität in Prag 1918-1945], Prag 2011.

${ }^{20}$ Vgl. Wolfgang Schievelbusch, Die Kultur der Niederlage. Der amerikanische Süden 1865, Frankreich 1871, Deutschland 1918, Berlin 2001.

${ }^{21}$ Vgl. Robert Gerwarth, The Vanquished. Why the First World War Failed to End, 1917-1923, London 2016.
} 
In dieser Hinsicht war auf den ersten Blick auch die Situation der Tschechoslowakei mit vielen Risiken verbunden. Die Republik war ein multiethnischer Staat. Jedoch nicht nur dies: In einigen Grenzregionen und im Osten der neuen Republik waren darüber hinaus die nationalen Identitäten oft höchst fließend und nur schwer greifbar. ${ }^{22}$ Umso mehr gewann in dieser Situation die Idee der siegreichen Nation eine wichtige Funktion für die Gestaltung und Stabilisierung des neuen demokratischen Staats. Ich werde diese These an drei Fallbeispielen illustrieren.

Obwohl in der Tschechoslowakei im Unterschied zu Deutschland oder Österreich die Gewalt von paramilitärischen Einheiten keine besondere Rolle spielte, verlief der Übergang von der Kriegszeit zur Nachkriegszeit keineswegs gewaltlos. Das Ausmaß der Nachkriegsgewalt in der Tschechoslowakei ist sogar mit dem in Österreich vergleichbar. ${ }^{23}$ Sie ging jedoch von anderen Akteuren aus, wurde in einem anderen Kontext verstanden und auch schnell eingedämmt. Dies betrifft vor allem die uniformierte Gewalt der Nachkriegszeit. In Prag wurden etwa Menschen von heimgekehrten Soldaten, meistens Angehörigen der Tschechoslowakischen Legionen, geohrfeigt, weil sie angeblich Deutsch gesprochen hatten. ${ }^{24} \mathrm{Im}$ Herbst 1920 kulminierte die Spannung in Böhmen in einer Welle nationaler tschechisch-deutscher Konflikte, in deren Verlauf Statuen und Denkmäler gestürzt, Minderheitenschulen angegriffen, deutsche Inschriften beseitigt wurden; in Prag besetzte ein von Legionären geführter tschechischer Mob gewaltsam das deutsche Theater. ${ }^{25}$

Immerhin blieb das Ausmaß der Gewalt im Westen der neuen Republik vergleichsweise begrenzt, brutalere Formen der Gewalt findet man nur selten. Die mehrere Jahrzehnte dauernde Erfahrung des deutsch-tschechischen nationalen Konflikts strukturierte den Raum und schuf ein System der nationalen Symbole und Narrative, auf welches man in einer krisenhaften Situation zurückgreifen und Gewalt in eine symbolische Ebene überführen konnte. Passanten in Prag, die Deutsch sprachen, wurden geohrfeigt und nicht ermordet. Die Gewalthandlung zielte auf die Rekodierung des öffentlichen Raums, nicht auf die Liquidation eines nationalen Feinds. ${ }^{26}$ Das nationale Siegesnarrativ half zugleich, das Gewalt-

${ }^{22}$ Vgl. Jeremy King, Budweisers into Czechs and Germans. A local history of Bohemian politics, 1848-1948, Princeton 2002; Pieter M. Judson, Guardians of the nation. Activists on the language frontiers of imperial Austria, Cambridge 2006, und Tara Zahra, Kidnapped Souls. National Indifference and the Battle for the Children in the Bohemian Lands, 1900-1948, Ithaca 2008.

${ }^{23}$ Vgl. Rudolf Kučera, Exploiting Victory, Sinking into Defeat. Uniformed Violence in the Creation of the New Order in Czechoslovakia and Austria, 1918-1922, in: Journal of Modern History 88 (2016), S. 827-855, hier S. 835 f.

${ }^{24}$ Vgl. Ota Konrád, Two Post-War Paths. Popular Violence in the Bohemian Lands and in Austria in the Aftermath of World War I, in: Nationalities Papers; online 27.10.2017: www.tandfonline.com/doi/full/10.1080/00905992.2017.1354362 [17.1.2018].

${ }^{25}$ Vgl. Peter Becher, Kulturpolitische Konfliktherde in der Ersten Republik. Der Streit um das Prager Ständetheater 1920 und die Prager Tonfilmaffäre 1930, in: Jörg K. Hönsch/Dušan Kováč (Hrsg.), Das Scheitern der Verständigung. Tschechen, Deutschen und Slowaken in der Ersten Republik (1918-1938), Essen 1994, S. 119-133, hier S. 122-127.

${ }^{26}$ Vgl. Konrád, Two Post-War Paths. 
potenzial der ehemaligen Mitglieder der Tschechoslowakischen Legion einzudämmen und sie in den Staat zu integrieren. ${ }^{27}$

Die Nachkriegsgewalt begann ab Herbst 1920 abzuklingen. Dies war, und damit komme ich zum zweiten Beispiel, durch die innenpolitische Stabilisierung möglich, für welche abermals die Kategorie der Nation eine große Rolle spielte. In Reaktion auf die innenpolitische Krise im Herbst 1920 - die nationalen Ausschreitungen und die Krise der Sozialdemokratie, welche in die Gründung der Kommunistischen Partei mündete ${ }^{28}$ - bildeten die Spitzenpolitiker der wichtigsten tschechischen politischen Parteien von links bis rechts den sogenannten Fünfer-Ausschuss, in welchem die maßgeblichen Entscheidungen fernab der parlamentarischen Kontrolle fielen. Daneben bildete sich um den Präsidenten, der eine einflussreiche Stellung innehatte, ein weiterer Zirkel, die sogenannte BurgGruppe. So entstanden zwei informelle Gremien, die trotz aller persönlichen, parteipolitischen und ideologischen Gegensätze eine starke stabilisierende Funktion hatten. ${ }^{29}$ Damit wurde unter anderem auch ermöglicht, den kurz nach 1918 aufgeflammten Antisemitismus einzudämmen, ${ }^{30}$ und vor allem das politische System zu stärken, welches bis zum Ende der 1930er Jahre den autoritären Tendenzen standhalten konnte. ${ }^{31}$

Ohne die Bejahung des tschechoslowakischen nationalen Staats und des gemeinsamen Interesses an seinem Weiterbestehen wäre jedoch die Zusammenarbeit nie möglich gewesen. Diese konsensorientierte politische Kultur wirkte integrierend, unter gewissen Vorbehalten konnte an ihr sogar ein Teil der sudetendeutschen Politik teilnehmen. Ab 1926 saßen in der Regierung auch Minister der „aktivistischen“, das heißt für die aktive Zusammenarbeit im Rahmen des tschechoslowakischen Staats aufgeschlossenen, sudetendeutschen Parteien. Eine unausgesprochene Voraussetzung stellte jedoch die Anerkennung der führenden Rolle der staatstragenden tschechoslowakischen Nation dar. ${ }^{32}$ Den vielen,

${ }^{27}$ Vgl. Ivan Šedivý, Zur Loyalität der Legionäre in der Ersten Tschechoslowakischen Republik, in: Martin Schulze Wessel (Hrsg.), Loyalitäten in der Tschechoslowakischen Republik 19181938. Politische, nationale und kulturelle Zugehörigkkeiten, München 2004, S. 141-152.

${ }^{28}$ Vgl. Antonín Klimek, Velké dějiny zemí koruny české [Große Geschichte der Länder der Böhmischen Krone], Bd. XIII: 1918-1929, Prag/Litomyšl 2000, S. 211-226.

${ }^{29}$ Zur Politik in der Tschechoslowakei vgl. Philip J. Howe/Thomas A. Lorman/Daniel E. Miller, The Creation of the Conditions for Consociational Democracy and Its Development in Interwar Czechoslovakia, in: Bohemia 56 (2016), S. 362-380; Andrea Orzoff, Battle for the Castle. The Myth of Czechoslovakia in Europe, 1914-1948, New York 2009, und Melissa Feinberg, Elusive Equality. Gender, Citizenship, and the Limits of Democracy in Czechoslovakia, 1918-1950, Pittsburgh 2006.

${ }^{30}$ Vgl. Michal Frankl/Miloslav Szabó, Budování státu bez antisemitismu? Násilí, diskurz loajality a vznik Československa [Aufbau des Staats ohne Antisemitismus? Gewalt, Loyalitätsdiskurs und Entstehung der Tschechoslowakei], Prag 2015.

${ }^{31}$ Vgl. Boris Barth, Europa nach dem Großen Krieg. Die Krise der Demokratie in der Zwischenkriegszeit 1918-1938, Frankfurt a. M./New York 2016, S. 163-165.

${ }^{32}$ Vgl. Jaroslav Kučera, Politický či přirozený národ? K pojetí národa v československém právním řádu meziválečného období [Politische oder natürliche Nation? Zur Deutung der Nation in der tschechoslowakischen Rechtsordnung der Zwischenkriegszeit], in: Český časopis historický 99 (2001), S. 548-568, und ders., Minderheit im Nationalstaat. 
vergleichsweise weitgehenden Zugeständnissen an die nationalen Minderheiten wurde daher eine klare Grenze gesetzt: Die sudetendeutschen Minister nahmen etwa an den wichtigsten informellen Verhandlungen nicht teil, es war auch unvorstellbar, dass ein Sudetendeutscher zum Verteidigungsminister, Außenminister oder Generalstabschef, geschweige zum Premierminister wurde.

Als letztes Beispiel für die Bedeutung der Kategorie Nation bei der Bildung des neuen Staats erwähne ich das Beispiel der Volkszählung. Im Februar 1921 fand die erste Volkszählung in der Tschechoslowakei statt, die schon im Vorfeld breit diskutiert und politisiert wurde. ${ }^{33}$ Im Unterschied zu den früheren Volkszählungen in der Monarchie wurde nicht mehr nach Umgangssprache gezählt. Jeder Bürger musste sich zu einer Nationalität bekennen. Darüber hinaus wurde es dem Staat beziehungsweise den Zählkommissaren erlaubt, in Streitfällen selbst über die Nationalität der Einzelnen nach „objektiven“ Kriterien - wie der Muttersprache - zu entscheiden. ${ }^{34}$ Die Volkszählung war daher nicht nur eine statistische Datenerhebung; sie ordnete, gestaltete und klärte die nationalen Verhältnisse und Identitäten im neuen Staat dadurch, dass jeder gezwungen wurde, national Farbe zu bekennen. Damit sollten die Bürger auch zu einer Selbstvergewisserung des neuen Staats beitragen.

In allen diesen Fällen wurde die Nation zum Prinzip der Stabilisierung des Staats, der Verwaltung und Kontrolle, zur Folie, durch welche der neue Staat gestaltet und organisiert wurde. Obwohl einerseits stabilisierend, führte eine solche Deutung zugleich zu neuen Widersprüchen. Die Nationalisierung im Sinne eines klaren Bekenntnisses zu der einen oder anderen Nation ermöglichte einerseits die politische Herrschaft, da sie den Raum übersichtlich strukturierte - sie war für die Schul-, Kultur- oder Sprachpolitik bedeutend -, zugleich rief sie jedoch neue Nationalismen hervor, die in Krisenzeiten für den Staat bedrohlich werden konnten. Eine nötige Kurskorrektur in Richtung der Modifikation eines Staats mit anerkannten nationalen Minderheiten hin zum Nationalitätenstaat oder sogar zum Staat einer politischen Nation kam zu spät oder war zu schwach ausgeprägt. ${ }^{35}$

${ }^{33}$ Vgl. Pavel Kladiwa u. a., Národnostní statistika v českých zemích 1880-1930. Mechanismy, problémy a důsledky národnostní klasifikace [Die Nationalitätenstatistik in den böhmischen Ländern. Mechanismen, Probleme und Folgen einer nationalen Klassifikation], 2 Bde., Prag 2016.

${ }^{34}$ Zit. nach Koeltzsch, Geteilte Kulturen, S. 42.

${ }^{35}$ Hier ist auch auf die - nicht wenigen - sudetendeutschen Antifaschisten hinzuweisen, welche sich in der Krisenzeit der 1930er Jahre aktiv hinter den tschechoslowakischen Staat stellten. Zum terroristischen Druck der nationalistischen bzw. schon nationalsozialistischen Umgebung auf solche pro-demokratische Kräfte im Frühling und Sommer 1938 vgl. Detlef Brandes, Die Sudetendeutschen im Krisenjahr 1938, München 2008. 


\section{Nation als Horizont des Denkens und Handelns}

Der durch die Eliten vermittelte siegreiche tschechoslowakische Nationsdiskurs gewann nach dem Ersten Weltkrieg eine verstärkte Bedeutung auch für die breiteren Massen der Bevölkerung, da er vermochte, dem Leiden an der Front und in der Heimat einen Sinn zu geben und den Raum, der durch die De-Legitimierung der alten Ordnung im Krieg entstanden worden war, zu füllen. So zeigen viele kleinere Alltagsbeispiele aus der unmittelbaren Nachkriegszeit, wie Menschen versuchten, die Kategorien dieses Elitendiskurses wie Nation, Demokratie, Republik, Freiheit, Neuanfang zu begreifen und sie in ihrem Handeln zu verwirklichen. Sie lehnten zum Beispiel das Eingreifen und Festnahmen durch die Polizei und Gendarmerie ab, mit der Begründung, jetzt herrsche die Freiheit. Nach einem Bericht des Innenministeriums vom Januar 1919 waren die Leute überzeugt, die Gendarmerie habe in der Republik kein Recht, Festnahmen durchzuführen. Das Wildern in einem Adelsgehege wurde damit erklärt, dass es „heute Freiheit und Teilung gibt, und daher auch wir Anspruch auf dieses Wild haben." 36

Die Antwort von unten auf den elitären tschechoslowakischen Nationsdiskurs in Form der Vorstellungen über eine gemeinsame Zukunft, Freiheit und Republik prägte auch die Demonstrationen im Mai 1919 gegen die Preissteigerung und den sogenannten Kettenhandel. Die Protestierenden trugen Schilder mit Sprüchen wie „Wir wollen eine Republik ohne Diebe“. Eine zentrale Rolle spielten dabei Gerechtigkeitsrituale. Die Demonstrierenden trugen improvisierte Holzgalgen, in deren Schlingen Händler und Bauern ihre Köpfe hineinlegen und schwören mussten, dass sie die Leute mit Waren und Lebensmitteln zu gerechten Preise versorgen würden. Diese Rituale setzten jedoch voraus, dass die Demonstrierenden eine Vorstellung der gemeinsamen aussichtsreichen Zukunft teilten: Vergebung und Besserung waren nämlich nur dann möglich, wenn man an eine gemeinsame Zukunft glaubte. ${ }^{37}$

Jedoch auch in diesen Fällen kann man von einer inneren Widersprüchlichkeit sprechen: Die in diesen Beispielen von unten her realisierte Verbindung zwischen Nation einerseits und ihrer Zukunftsorientierung, Vergebung, Freiheit, Republikanismus andererseits konnten nur dann existieren, wenn diese Kategorien auch gültig blieben. Nach dem Schock im September 1938 war es jedoch nur die Nation im ethnischen Sinne, die übriggeblieben war: aus der Pro-Westlichkeit wurde der westliche Verrat, Freiheit und Demokratie machten schnell der Diktatur der kurzlebigen Zweiten Republik Platz, an Stelle der Toleranz blühte der Antisemitismus wieder auf, aus der Fortschrittlichkeit und dem Zukunftsglauben wurde eine konservative Rückkehr zu den Wurzeln des tschechischen nation building im 19.

\footnotetext{
${ }^{36}$ Das Thema samt diesen Beispielen ist ausführlicher dargestellt in dem Buch von Ota Konrád/Rudolf Kučera, Cesty z apokalypsy. Fyzické násilí v pádu a obnově střední Evropy 19141922 [Wege aus der Apokalypse. Physische Gewalt im Fall und Wiederaufbau von Zentraleuropa 1914-1922], im Druck. Vgl. weiterhin Václav Šmidrkal, Fyzické násilí, státní autorita a trestní právo v českých zemích 1918-1923 [Physische Gewalt, Staatsautorität und Strafrecht in den Böhmischen Ländern 1918-1923], in: Český časopis historický 114 (2016), S. 89-115.

37 Vgl. Konrád, Two Post-War Paths.
} 
Jahrhundert, was die tschechische Kultur auch in den folgenden Jahren stark prägte. ${ }^{38}$

\section{Zusammenfassung und Ausblick}

Über München als Ende der Ersten Republik kursierten fast von Anfang an unterschiedliche Erklärungen, welche auch in den fachhistorischen Deutungen zu finden sind. Grob gesehen bewegen sie sich zwischen zwei gegensätzlichen Polen. Nach einer Deutung unterlag die beinah vorzügliche und Ende der 1930er Jahre auch einzige Demokratie in Zentraleuropa einer Übermacht, wobei sie zugleich infolge des kurzsichtigen Taktierens ihrer westlichen Verbündeten verraten wurde. ${ }^{39}$ Nach der anderen Deutung, welche vorwiegend in der sudetendeutschen Literatur zu finden ist, war jedoch der Hauptgrund des Scheiterns der Republik nicht die Intervention von außen. Diese war vielmehr nur ein letzter Schlag für einen innerlich schon maroden, künstlich geschaffenen Staat, der von Anfang an geradezu eine „Fehlkonstruktion" ${ }^{\text {"40 }}$ war und sich vor allem im Falle seiner Nationalitätenpolitik von den angrenzenden autoritären Regimen nicht grundsätzlich unterschied.

Der Grund für den Niedergang der Ersten Republik war jedoch weder der Druck von außen, welcher einen funktionierenden, innerlich kohärenten, vorzüglich demokratischen Staat zerbrochen hätte, noch der innere Zusammenbruch eines künstlichen, seine nationalen Minderheiten unterdrückenden Staatsgebildes. Das Problem lag eher darin, dass die ungünstige internationale Situation keinen Raum übrig ließ, um zu zeigen, ob dieser Staat fähig war, der vielen inneren Widersprüche Herr zu werden und seine Existenzberechtigung unter Beweis zu stellen. Die Erste Republik hat es in den 20 Jahren ihrer Existenz nicht geschafft, ihr eigenes Projekt voll zu entfalten.

Gerade aus diesen Gründen ist ihr demokratisches Erbe nicht eindeutig, und es ist schwer daran anzuknüpfen. In jedem Fall gilt: Die inneren Probleme dieser Gesellschaft sind ohne die weiteren transnationalen und internationalen Zusammenhänge nicht zu begreifen. Diese fast banale Feststellung stellt immerhin eine Herausforderung für die Geschichtsschreibung über die Zwischenkriegszeit dar: Eine Geschichte der Tschechoslowakei, wie sie bisher allzu oft nur aus der tschechischen oder sogar Prager Perspektive geschrieben wurde, hat wenig Sinn. Gera-

${ }^{38}$ Vgl. Peter Bugge, Czech Democracy 1918-1938. Paragon or Parody?, in: Bohemia 47 (2007), S. 3-28. Zur Deutung der Nation im Protektorat Böhmen und Mähren vgl. Chad Bryant, Prague in Black. Nazi Rule and Czech Nationalism, Cambridge/London 2007. Zur Kulturpolitik im Protektorat vgl. Volker Mohn, NS-Kulturpolitik im Protektorat Böhmen und Mähren. Konzepte, Praktiken, Reaktionen, Essen 2014.

${ }^{39}$ Vgl. Jindřich Dejmek, Edvard Beneš. Politická biografie českého demokrata. Část druhá. Prezident republiky a vůdce národního odboje (1935-1948) [Edvard Beneš. Politische Biografie eines tschechischen Demokraten. Teil II: Präsident der Republik und Führer des nationalen Widerstandes (1935-1948)], Prag 2008.

${ }^{40}$ Vgl. Hellmut Diwald/Rolf-Josef Eibicht (Hrsg.), Die Tschechoslowakei. Das Ende einer Fehlkonstruktion. Die sudetendeutsche Frage bleibt offen, Berg ${ }^{2} 1993$. 
de die Tschechoslowakei der Zwischenkriegszeit ist eher als Knotenpunkt der allgemeineren (zentral-)europäischen Entwicklungen zu deuten, welche hier vielleicht eine spezifische Färbung bekamen, oft sogar abgelehnt wurden (um stattdessen eine vermeintlich echte, eigene Tradition hervorzuheben), die jedoch stets ohne dieses Netzwerk nicht verstehbar ist. Darüber hinaus ermöglicht eine Deutung, die die Tschechoslowakei der Zwischenkriegszeit als ein unvollendetes Projekt sieht, dass sie nicht mehr - wie das bis heute fast ausnahmslos geschieht - von ihrem Ende her betrachtet wird. Dies wird hoffentlich auch die gegenwärtige Diskussion über die Erste Republik und die Erinnerung an sie prägen, welche gerade im Jubiläumsjahr 2018 allerorten präsent sein wird. ${ }^{41}$

\footnotetext{
${ }^{41}$ Diese Studie entstand im Rahmen der Forschungsprojekte „Charles University Research Centre program No. 9“ und „Violence in Central Europe During and in the Aftermath of the World War I. Austrian and Czech Lands in Compariso" (GAČR 14-14612S).
} 


\section{DE}

\section{QUELLEN ZU DEN BEZIEHUNGEN ZWISCHEN DER BUNDESREPUBLIK DEUTSCHLAND UND UNGARN $1949-1990$}

\section{NEUE EDITION}

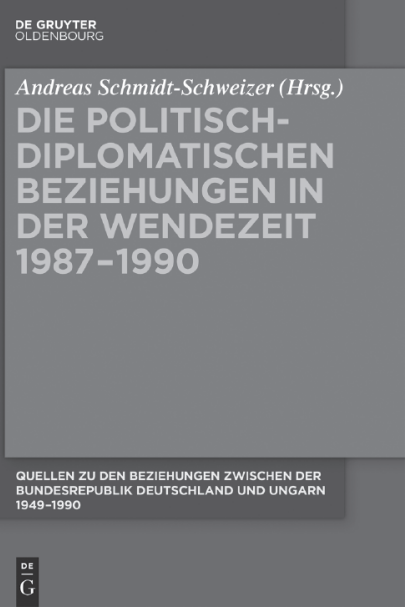

Andreas Schmidt-Schweizer (Hrsg.)

Band 3

\section{DIE POLITISCH-DIPLOMATISCHEN BEZIEHUNGEN} IN DER WENDEZEIT 1987-1990

2017, XVI, 744 S.

Geb. $€ 169,95$ [D]

ISBN 978-3-11-048623-0

eBook $€ 169,95$ [D]

PDF ISBN 978-3-11-048889-0

ePUB ISBN 978-3-11-048644-5

\section{IN VORBEREITUNG:}

\section{Band 1 1949-1973}

Band 2 1973-1987

Die dreibändige Edition dokumentiert und analysiert die Entwicklung der bilateralen Beziehungen zwischen der Bundesrepublik Deutschland und Ungarn von 1949-1990. Der Blick richtet sich vor allem auf die zwischenstaatlichen Wirtschaftsbeziehungen und die politisch-diplomatischen Kontakte. Den herausragenden Schriftquellen gehen ausführliche monografische Einführungen voraus.

Band 3 thematisiert die Wendejahre von 1987-1990 vor dem Hintergrund des ungarischen Reform- und Transformationsprozesses und der deutschen Wiedervereinigung. Chronologie, Dokumentenverzeichnis, Kurzbiografien der wichtigsten politischen Akteure und ein umfangreiches Register erschließen den Band.

Andreas Schmidt-Schweizer ist wissenschaftlicher Mitarbeiter am Institut für Geschichtswissenschaften des Zentrums für Humanwissenschaften der Ungarischen Akademie der Wissenschaften, Budapest. 